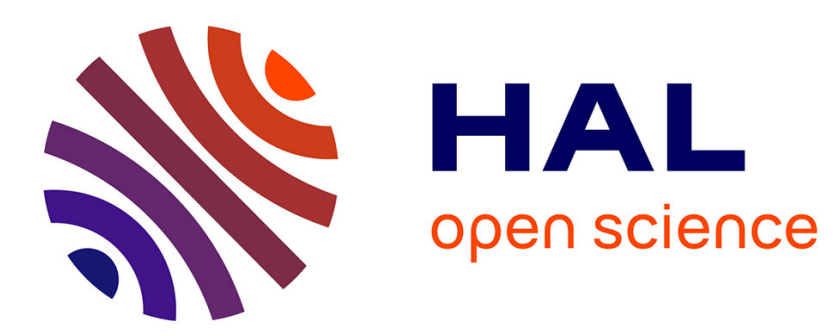

\title{
Diagrammatic logic applied to a parameterization process
}

Cesar Dominguez, Dominique Duval

\section{To cite this version:}

Cesar Dominguez, Dominique Duval. Diagrammatic logic applied to a parameterization process. Mathematical Structures in Computer Science, 2010, 20 (4), pp.639-654. 10.1017/S0960129510000150 . hal-00411069

\section{HAL Id: hal-00411069 \\ https://hal.science/hal-00411069}

Submitted on 25 Aug 2009

HAL is a multi-disciplinary open access archive for the deposit and dissemination of scientific research documents, whether they are published or not. The documents may come from teaching and research institutions in France or abroad, or from public or private research centers.
L'archive ouverte pluridisciplinaire HAL, est destinée au dépôt et à la diffusion de documents scientifiques de niveau recherche, publiés ou non, émanant des établissements d'enseignement et de recherche français ou étrangers, des laboratoires publics ou privés. 


\title{
Diagrammatic logic applied to a parameterization process
}

\author{
César Domínguez * Dominique Duval ${ }^{\dagger}$
}

August 25., 2009

\begin{abstract}
This paper provides an abstract definition of some kinds of logics, called diagrammatic logics, together with a definition of morphisms and of 2-morphisms between diagrammatic logics. The definition of the 2-category of diagrammatic logics rely on category theory, mainly on adjunction, categories of fractions and limit sketches. This framework is applied to the formalization of a parameterization process. This process, which consists in adding a formal parameter to some operations in a given specification, is presented as a morphism of logics. Then the parameter passing process, for recovering a model of the given specification from a model of the parameterized specification and an actual parameter, is seen as a 2-morphism of logics.
\end{abstract}

\section{Introduction}

This paper provides an introduction to the framework of diagrammatic logics with an application to the formalization of a parameterization process.

The framework of diagrammatic logics is presented in section 2. It stems from Duval 2003, Duval 2007, where the aim was to get an abstract definition of logics, with relevant notions of models and proofs, together with a good notion of morphism between logics: we were looking for kinds of logics for dealing with computational effects and for morphisms for expressing the meaning of the effects into more usual logics. This work is based on adjunction Kan 1958 and categories of fractions [Gabriel and Zisman 1967] with an additional level of abstraction provided by limit sketches Ehresmann 1968], which leads to a notion of entailment apparented to Makkai 1997. Our point of view is more abstract than the institutions Goguen and Burstall 1984, see \begin{tabular}{|l|l|l|}
\hline Duval 2003 for a comparison. This new paper does not depend on Duval 2003, Duval 2007. \\
\hline
\end{tabular}

On the other hand, the EAT and Kenzo software systems have been developed by F. Sergeraert for symbolic computation in algebraic topology Rubio et al. 2007, Dousson et al. 1999]. The data types used in EAT and Kenzo have been specified through a parameterization process in Domínguez et al. 2006, Domínguez et al. 2007, which is described in Lambán et al. 2003 in terms of object-oriented technologies like hidden algebras Goguen and Malcolm 2000 or coalgebras [Rutten 2000. The parameterization process consists in adding a formal parameter to some operations in a given specification. It is followed by the parameter passing process, which recovers a model of the given specification from any model of the parameterized specification and any actual parameter. A first attempt to use diagrammatic logics in order to formalize this parameterization process is given in Domínguez et al. 2005. In section 3 we present a simple formalization of the parameterization and parameter passing processes as a morphism and a 2-morphism of diagrammatic logics, respectively. The focus in this application is on the models, but in Dumas et al. 2009] another kind of application is studied, where proofs in a diagrammatic logic play an important role.

Most categorical notions used in this paper can be found in Mac Lane 1998 or Barr and Wells 1999. For simplicity, we omit most size issues and we do not always distinguish between equivalent categories. The

\footnotetext{
* Departamento de Matemáticas y Computación, Universidad de La Rioja, Edificio Vives, Luis de Ulloa s/n, E-26004 Logroño, La Rioja, Spain, cesar.dominguez@unirioja.es.

†Laboratoire Jean Kuntzmann, Université de Grenoble, 51 rue des mathématiques, BP 53, F-38041 Grenoble Cédex 9, France, Dominique.Duval@imag.fr.
} 
class of morphisms from $X$ to $Y$ in a category $\mathbf{C}$ is denoted $\mathbf{C}[X, Y]$. A graph means a directed multigraph, and in order to distinguish between various kinds of structures with an underlying graph we speak about the objects and morphisms of a category, the types and terms of a theory or a specification and the points and arrows of a limit sketch. The diagrammatic logics which are considered in this paper are the equational logic and several apparented logics. However diagrammatic logics can be much richer, for instance first-order logic as well as simple lambda calculus and logics with induction or coinduction can be seen as diagrammatic logics.

\section{Diagrammatic logics}

The 2-category of diagrammatic logics and its related notions are defined in sections 2.1, 2.2 and 2.3, then the diagrammatic equational logic is described in section 2.4 .

\subsection{Limit sketches}

There are several definitions of limit sketches (also called projective sketches), all of them are such that a limit sketch generates a category with limits Coppey and Lair 1984, Barr and Wells 1999. While a category with limits is a graph with identities, compositions, limit cones and tuples, satisfying a bunch of axioms, we define a limit sketch $\mathbf{E}$ as a graph with potential identities, compositions, limit cones and tuples, which become real features in the generated category with limits $C(\mathbf{E})$. For instance a point $X$ in $\mathbf{E}$ may have a potential identity, this is an arrow $i d_{X}: X \rightarrow X$ in $\mathbf{E}$ which becomes the identity morphism at the object $X$ in $C(\mathbf{E})$. As another instance, a diagram in $\mathbf{E}$ may have a potential limit cone, which becomes a limit cone in $C(\mathbf{E})$. Potential features are not required to satisfy any axiom in $\mathbf{E}$. In addition, for the simplicity of notations, we assume that each potential feature is unique: a point has at most one potential identity, a diagram has at most one potential limit cone, and so on.

A morphism of limit sketches $\mathbf{e}: \mathbf{E}_{1} \rightarrow \mathbf{E}_{2}$ is a graph morphism which maps the potential features of $\mathbf{E}_{1}$ to potential features of $\mathbf{E}_{2}$. This forms the category of limit sketches. A realization (or loose model) of a limit sketch $\mathbf{E}$ with values in a category $\mathbf{C}$ is a graph morphism which maps the potential features of $\mathbf{E}$ to real features of $\mathbf{C}$. A morphism of realizations is (an obvious generalization of) a natural transformation. This gives rise to the category $\operatorname{Real}(\mathbf{E}, \mathbf{C})$ of realizations of $\mathbf{E}$ with values in $\mathbf{C}$, denoted $\operatorname{simply} \operatorname{Real}(\mathbf{E})$ when $\mathbf{C}$ is the category of sets. The category $\operatorname{Real}(\mathbf{E})$ has colimits and we will use the fact that left adjoint functors preserve colimits.

The Yoneda contravariant realization $\mathcal{Y}_{\mathbf{E}}$ of a limit sketch $\mathbf{E}$ takes its values in $\operatorname{Real}(\mathbf{E})$. It is defined as $\mathcal{Y}_{\mathbf{E}}(E)=P(\mathbf{E})[E,-]$ where $P(\mathbf{E})$ is the prototype of $\mathbf{E}$, which means, the category generated by $\mathbf{E}$ such that every potential feature of $\mathbf{E}$ becomes a real feature of $P(\mathbf{E})$. Thanks to $\mathcal{Y}_{\mathbf{E}}$, up to contravariance the limit sketch $\mathbf{E}$ can be identified to a part of $\operatorname{Real}(\mathbf{E})$ which will be called the elementary part of $\operatorname{Real}(\mathbf{E})$ (with respect to $\mathbf{E})$ and denoted $\operatorname{Real}_{e l}(\mathbf{E})$. It is a graph with distinguished features, defined as the identities, compositions, colimits and cotuples which are the images of the potential features of E. A fundamental property is that the elementary part of $\operatorname{Real}(\mathbf{E})$ is dense in $\operatorname{Real}(\mathbf{E})$ : every realization or morphism of realizations of $\mathbf{E}$ can be obtained by colimits and cotuples from $\operatorname{Real}_{e l}(\mathbf{E})$. Moeover, a fundamental theorem due to Ehresmann states that every morphism of limit sketches $\mathbf{e :} \mathbf{E}_{1} \rightarrow \mathbf{E}_{2}$ gives rise to an adjunction $F_{\mathbf{e}} \dashv G_{\mathbf{e}}$ where the right adjoint $G_{\mathbf{e}}$ is the precomposition with e Ehresmann 1968:

$$
\operatorname{Real}\left(\mathbf{E}_{1}\right) \underset{G_{\mathbf{e}}}{\frac{F_{\mathbf{e}}}{\perp}} \operatorname{Real}\left(\mathbf{E}_{2}\right)
$$

Then the functor $F_{\mathbf{e}}$ contravariantly extends e via the Yoneda contravariant realizations, in the sense that there is a natural isomorphism:

$$
F_{\mathbf{e}} \circ \mathcal{Y}_{\mathbf{E}_{1}} \cong \mathcal{Y}_{\mathbf{E}_{2}} \circ \mathbf{e}
$$

A locally presentable category Gabriel and Ulmer 1971 is a category $\mathbf{C}$ which is equivalent to the category of set-valued realizations of a limit sketch $\mathbf{E}$, then $\mathbf{E}$ is called a limit sketch for the category $\mathbf{C}$. In addition, 
we define a locally presentable functor as a functor $F: \mathbf{C}_{1} \rightarrow \mathbf{C}_{2}$ which is the left adjoint to the precomposition with some morphism of limit sketches $\mathbf{e}$, so that $\mathbf{C}_{1}$ and $\mathbf{C}_{2}$ are locally presentable categories. Then $\mathbf{e}$ is called a morphism of limit sketches for the functor $F$.

\subsection{Diagrammatic logic: models and proofs}

The framework of diagrammatic logics stems from Duval 2003, Duval 2007.

Definition 2.1 A diagrammatic logic is a locally presentable functor $L$ such that its right adjoint $R$ is full and faithful.

The fact that $R$ is full and faithful is equivalent to the fact that the counit natural transformation $\varepsilon: L \circ R \Rightarrow I d$ is an isomorphism. According to Gabriel and Zisman 1967, it is also equivalent to the fact that $L$ is a localization, up to an equivalence of categories: it consists of adding inverse morphisms for some morphisms, constraining them to become isomorphisms. Let us consider a diagrammatic logic $L$ :

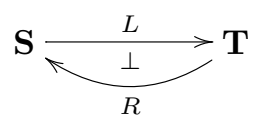

Definition 2.1 also means that $R$ defines an isomorphim from $\mathbf{T}$ to its image, which is a reflective subcategory of $\mathbf{S}$

Definition 2.2 The categories $\mathbf{S}$ and $\mathbf{T}$ are the category of specifications and the category of theories, respectively, of the diagrammatic logic $L$. A specification $\Sigma$ presents a theory $\Theta$ if $\Theta$ is isomorphic to $L(\Sigma)$. Two specifications are equivalent if they present the same theory.

The fact that $R$ is full and faithful means that every theory $\Theta$, when seen as a specification $R(\Theta)$, presents itself. With the next definition, we claim that every model of a specification takes its values in some theory.

Definition 2.3 A (strict) model $M$ of a specification $\Sigma$ in a theory $\Theta$ is a morphism of theories $M: L \Sigma \rightarrow \Theta$ or equivalently (thanks to the adjunction) a morphism of specifications $M: \Sigma \rightarrow R \Theta$.

It follows that equivalent specifications have the same models. A model $M$ of $\Sigma$ in $\Theta$ is sometimes called an oblique morphism, it is denoted $M: \Sigma \rightarrow \Theta$. Whenever in addition $\mathbf{S}$ and $\mathbf{T}$ are 2-categories with a natural isomorphism between $\mathbf{T}[L \Sigma, \Theta]$ and $\mathbf{S}[\Sigma, R \Theta]$, then $\mathbf{T}[L \Sigma, \Theta]$ is the category of models of $\Sigma$ in $\Theta$, denoted $L[\Sigma, \Theta]$. Otherwise, $L[\Sigma, \Theta]$ is simply the discrete category with the models of $\Sigma$ in $\Theta$ as objects.

Definition 2.4 An entailment is a morphism $\tau$ in $\mathbf{S}$ such that $L \tau$ is invertible in $\mathbf{T}$.

A similar notion can be found in Makkai 1997. Two specifications which are related by entailments are equivalent.

Definition 2.5 An instance $\rho$ of a specification $\Sigma$ in a specification $\Sigma_{1}$ is a cospan in $\mathbf{S}$ made of a morphism $\sigma: \Sigma \rightarrow \Sigma_{1}^{\prime}$ and an entailment $\tau: \Sigma_{1} \rightarrow \Sigma_{1}^{\prime}$. It is also called a fraction with numerator $\sigma$ and denominator $\tau$, and it is denoted $\rho=\tau \backslash \sigma: \Sigma \rightarrow \Sigma_{1}$.

Let us illustrate an instance $\rho=\tau \backslash \sigma$ of $\Sigma$ in $\Sigma_{1}$ as:

$$
\Sigma \stackrel{\sigma}{\longrightarrow} \Sigma_{1}^{\prime} \underset{\tau}{\stackrel{---}{\longleftarrow}} \Sigma_{1}
$$

this provides easily a diagram in the category $\mathbf{S}$, by omitting the dotted arrow, and a diagram in the category $\mathbf{T}$, by making the dotted arrow a solid one, inverse to $L \tau$ :
in $\mathbf{S}:$

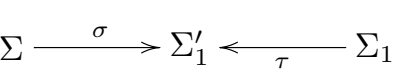
in $\mathbf{T}: \quad L \Sigma \stackrel{L \sigma}{\longrightarrow} L \Sigma_{1}^{\prime} \stackrel{(L \tau)^{-1}}{\longleftarrow} L \Sigma_{1}$ 
Since the category $\mathbf{S}$ has colimits and since the composition of entailments is an entailment, the instances can be composed in the usual way as cospans, thanks to pushouts. This forms the bicategory of instances of the logic, denoted $\mathbf{S}_{\mathbf{2}}$. Let $\rho=\tau \backslash \sigma: \Sigma \rightarrow \Sigma_{1}$ in $\mathbf{S}_{\mathbf{2}}$, then we define $L \rho=(L \tau)^{-1} \circ L \sigma: L \Sigma \rightarrow L \Sigma_{1}$ in $\mathbf{T}$. The instances are better suited than the morphisms of specifications for presenting the morphisms of theories, because for every morphism of theories $\theta: L \Sigma \rightarrow L \Sigma_{1}$ there is an instance $\rho$ such that $L \rho=\theta$. Since $L$ is a localization, the quotient category of the bicategory $\mathbf{S}_{\mathbf{2}}$ is equivalent to $\mathbf{T}$.

Definition 2.6 An inference system for a diagrammatic logic $L$ is a morphism of limit sketches $\mathbf{e}: \mathbf{E}_{S} \rightarrow \mathbf{E}_{T}$ for the locally presentable functor $L$.

Thanks to the Yoneda contravariant realization, the morphism e has properties similar to the functor $L$. In particular, e can be chosen so as to consist of adding inverse arrows for some collection of arrows in $\mathbf{E}_{S}$; see [Duval 2003, theorem 3.13] for a systematic construction of e. The next definitions depend on the choice of an inference system $\mathbf{e}: \mathbf{E}_{S} \rightarrow \mathbf{E}_{T}$ for $L$; more details are given in [Duval 2007].

Definition 2.7 An inference rule $r$ with hypothesis $H$ and conclusion $C$ is a span in $\mathbf{E}_{S}$, made of two morphisms $t: H^{\prime} \rightarrow H$ and $s: H^{\prime} \rightarrow C$ such that $\mathbf{e}(t)$ is invertible in $\mathbf{E}_{T}$. It is also called a fraction with numerator $s$ and denominator $t$, and it is denoted $r=s / t: H \rightarrow C$.

With this definition we claim that an inference rule with hypothesis $H$ and conclusion $C$ can be seen, via the Yoneda contravariant realization, as an instance of $\mathcal{Y}(C)$ in $\mathcal{Y}(H)$. So, we can define an inference step simply as a composition of fractions, which means, as a pushout in the category $\mathbf{S}$.

Definition 2.8 Given an inference rule $r=s / t: H \rightarrow C$ and an instance $\kappa: \mathcal{Y}(H) \rightarrow \Sigma$ of the hypothesis $\mathcal{Y}(H)$ in a specification $\Sigma$, the corresponding inference step provides the instance $\kappa \circ \mathcal{Y}(r): \mathcal{Y}(C) \rightarrow \Sigma$ of the conclusion $\mathcal{Y}(C)$ in $\Sigma$.

Definition 2.9 A proof (or derivation, or derived rule) is the description of a fraction in $\mathbf{S}_{\mathbf{2}}$ in terms of inference rules (thanks to composition and cotuples).

Typically, by deriving $\rho=\tau \backslash i d_{\sigma}$ for a given morphism $\tau: \Sigma_{1} \rightarrow \Sigma$, we get the property that $\tau$ is an entailment. For instance, in equational logic, let $\tau$ be the inclusion of a given specification $\Sigma_{1}$ into the specification $\Sigma$ made of $\Sigma_{1}$ together with an equation $f=g$ made of two terms $f, g$ in $\Sigma_{1}$; then $\tau$ is an entailment if and only if the equation $f=g$ holds in the theory presented by $\Sigma_{1}$.

\subsection{The 2-category of diagrammatic logics}

Definition 2.10 A morphism of logics $F: L_{1} \rightarrow L_{2}$ is a pair of locally presentable functors $\left(F_{S}, F_{T}\right)$ together with a natural isomorphism $F_{T} \circ L_{1} \cong L_{2} \circ F_{S}$.

This means that there are inference systems $\mathbf{e}_{1}$ and $\mathbf{e}_{2}$ for $L_{1}$ and $L_{2}$ respectively, and morphisms of limit sketches $\mathbf{e}_{S}$ and $\mathbf{e}_{T}$ for $F_{S}$ and $F_{T}$ respectively, which form a commutative square of limit sketches:
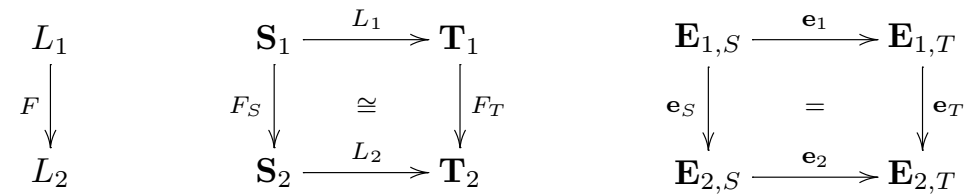

Using the Yoneda contravariant realization, a morphism of logics $F: L_{1} \rightarrow L_{2}$ can be determined by any graph morphism on $\mathbf{S}_{1, e l}$ (the elementary part of $\mathbf{S}_{1}$ with respect to $\mathbf{E}_{1}$ ) with values in $\mathbf{S}_{2}$ preserving the distinguished features of $\mathbf{S}_{1, e l}$ and the entailments of $L_{1}$. Some morphisms of logics are easier to describe at the sketch level (as the undecoration morphism in section 3.1) while others are easier to describe at the logic level (as the parameterization morphism in section 3.2). The next result is a straightforward application of adjunction. 
Proposition 2.11 Given a morphism of logics $F: L_{1} \rightarrow L_{2}$ and the corresponding adjunctions $F_{T} \dashv G_{T}$ between theories and $F_{S} \dashv G_{S}$ between specifications, for each specification $\Sigma_{1}$ of $L_{1}$ and each theory $\Theta_{2}$ of $L_{2}$ the adjunctions provide an isomorphism, natural in $\Sigma_{1}$ and $\Theta_{2}$, between the categories of models:

$$
L_{1}\left[\Sigma_{1}, G_{T}\left(\Theta_{2}\right)\right] \cong L_{2}\left[F_{S}\left(\Sigma_{1}\right), \Theta_{2}\right] .
$$

Definition 2.12 A 2-morphism of logics $\ell: F \Rightarrow F^{\prime}: L_{1} \rightarrow L_{2}$ is a pair of natural transformations $\left(\ell_{S}, \ell_{T}\right)$ where $\ell_{S}: F_{S} \Rightarrow F_{S}^{\prime}: \mathbf{S}_{1} \rightarrow \mathbf{S}_{2}$ and $\ell_{T}: F_{T} \Rightarrow F_{T}^{\prime}: \mathbf{T}_{1} \rightarrow \mathbf{T}_{2}$ are such that $\ell_{T} \circ L_{1}=L_{2} \circ \ell_{S}$.

Given a morphism of logics $F=\left(F_{S}, F_{T}\right)$ or a 2-morphism of logics $\ell=\left(\ell_{S}, \ell_{T}\right)$, we will usually omit the subscripts $S$ and $T$.

The diagrammatic logics together with their morphisms and 2-morphisms form a 2-category. By focusing on theories we get a functor from the 2-category of diagrammatic logics to the 2-category of categories. The other parts of the logic (the category of specifications, the adjunction, and the inference system) provide a way to answer some issues about theories, typically whether some morphisms of theories are invertible.

\subsection{The diagrammatic equational logic}

The equational logic provides a fundamental example of a diagrammatic logic. As usual in categorical logic (see [Pitts 2000]), the equational theories are defined as the categories with chosen finite products; with the functors which preserve the chosen finite products they form a category $\mathbf{T}_{e q}$. Similarly (see Lellahi 1989, Barr and Wells 1999, Wells 1993), the equational specifications are defined as the finite product sketches, which means, the limit sketches (as in section 2.1) such that their potential limits are only potential products; with the morphisms of finite product sketches they form a category $\mathbf{S}_{e q}$. Since all finite products may be recovered from binary products and a terminal type, we restrict the arity of products to either 2 or 0 . We will often omit the word "equational". Every theory $\Theta$ can be seen as a specification $R_{e q} \Theta$ and every specification $\Sigma$ generates, or presents, a theory $L_{e q} \Sigma$. This corresponds to an adjunction:

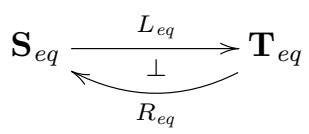

The category of sets with the cartesian products as chosen products forms an equational theory denoted Set. By default the models of an equational specification $\Sigma$ are the models of $\Sigma$ in Set, called the set-valued models of $\Sigma$. It is a classical exercise to build limit sketches for $\mathbf{T}_{e q}$ and $\mathbf{S}_{e q}$, then it is easy to check that $L_{e q}$ is a diagrammatic logic. A simplified description is given now, see Domínguez and Duval 2009 for a detailed construction. The starting point is the limit sketch for graphs $\mathbf{E}_{g r}$, where the points Type and Term stand for the sets of vertices (or types) and edges (or terms) and the arrows dom and codom for the functions source (or domain) and target (or codomain):

$$
\text { Type } \lesssim \frac{\text { dom }}{\text { codom }} \text { Term }
$$

Figure 1 presents the main part of the graph underlying $\mathbf{E}_{e q, S}$, in addition there are potential limits, including the specification of potential monomorphisms, and equalities of arrows. We have represented this graph in such a way that the bottom line, which is made of $\mathbf{E}_{g r}$ with potential limits and tuples, is equivalent to $\mathbf{E}_{g r}$. The point Type has been duplicated for readability, and the point Unit is a potential terminal type, interpreted as a singleton.

- The point Comp stands for the set of pairs of composable terms, the arrow $i$ for the inclusion into the set of pairs of consecutive terms and comp for $(f, g) \mapsto g \circ f$.

- The point Selid stands for the set of types with a potential identity, the arrow i0 for the inclusion and selid for $X \mapsto i d_{X}$. 
- The point 2-Prod stands for the set of pairs of types with a potential binary product, the arrow $j$ for the inclusion into the set of pairs of types and 2-prod for $\left(Y_{1}, Y_{2}\right) \mapsto\left(p r_{i}: Y_{1} \times Y_{2} \rightarrow Y_{i}\right)_{i=1,2}$.

- The point 2-Tuple stands for the set of binary cones with a potential binary tuple, the arrow $\mathrm{k}$ for the inclusion into the set of binary cones, 2-base' for recovering the base $\left(f_{i}: X \rightarrow Y_{i}\right)_{i=1,2} \mapsto\left(Y_{1}, Y_{2}\right)$, and 2-tuple stands for the construction of the potential binary tuple $\left(f_{i}: X \rightarrow Y_{i}\right)_{i=1,2} \mapsto\left\langle f_{1}, f_{2}\right\rangle: X \rightarrow$ $Y_{1} \times Y_{2}$.

- The point 0-Prod stands for the set of potential terminal types, the arrow $j 0$ for the injection (ensuring that there is at most one terminal type) and 0 -prod for the selection of the potential terminal type (if any).

- The point 0-Tuple stands for the set of types with a potential collapsing term (or nullary tuple), the arrow $\mathrm{k} 0$ for the inclusion into the set of types, 0 -base' for recovering the potential terminal type and 0 -tuple stands for the construction of the potential collapsing term $X \mapsto\langle\rangle_{X}: X \rightarrow 1$.

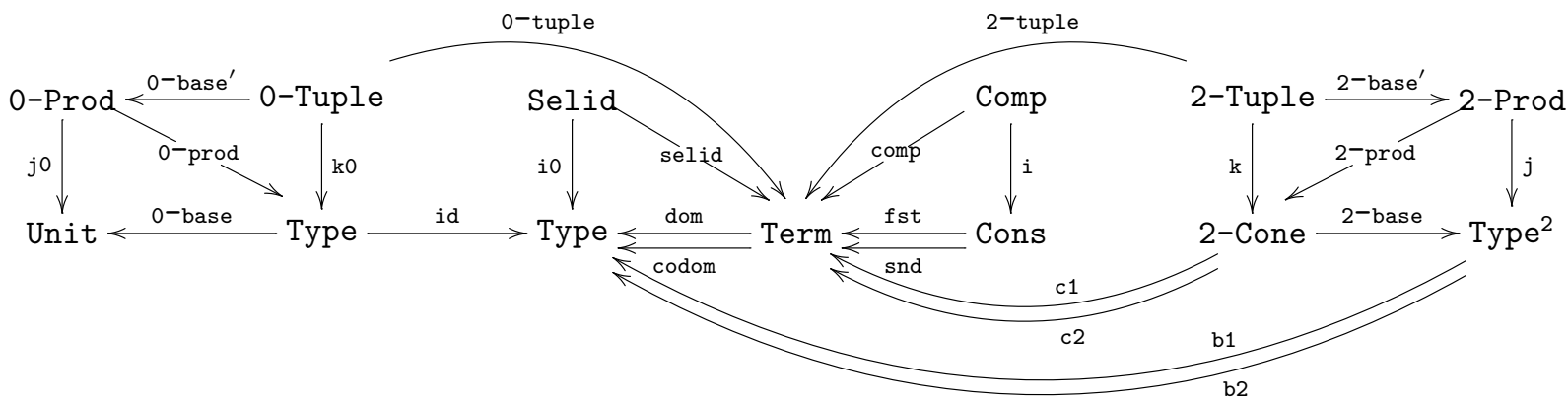

Figure 1: The graph underlying $\mathbf{E}_{e q, S}$

A limit sketch $\mathbf{E}_{e q, T}$ for equational theories is obtained from $\mathbf{E}_{e q, S}$ by choosing the entailments and mapping them to equalities, the corresponding morphism is the diagrammatic equational logic $L_{e q}$. Figure 2 provides the correspondence between the usual rules of equational logic and the diagrammatic inference rules, as fractions. Since only a part of $\mathbf{E}_{e q, S}$ is considered, some rules are missing, it is an exercise to enlarge $\mathbf{E}_{e q, S}$ so as to get them.

It should be noted that in this definition of the equational theories and specifications, the equations are identities of terms; a more subtle point of view, where the equations in a theory form a congruence, can be found in Domínguez and Duval 2009.

\section{A parameterization process}

Several variants of the diagrammatic equational logic, related by morphisms, are defined in section 3.1 . The parameterization process and the parameter passing process are formalized in sections 3.2 and 3.3 , respectively.

\subsection{Some diagrammatic logics}

The theories of the parameterized equational logic $L_{A}$ are the equational theories together with a distinguished type, called the type of parameters and usually denoted $A$. The specifications are the equational specifications with maybe a distinguished type $A$. The inclusion of limit sketches determines a morphism of $\operatorname{logics} F_{A}: L_{e q} \rightarrow L_{A}$. 


\begin{tabular}{|c|c|c|}
\hline name & rule & fraction \\
\hline composition & $\frac{f: X \rightarrow Y \quad g: Y \rightarrow Z}{g \circ f: X \rightarrow Z}$ & Cons $\bar{z}-->$ Comp $\stackrel{\text { comp }}{\longrightarrow}$ Term \\
\hline identity & $\frac{X}{i d_{X}: X \rightarrow X}$ & Type $\overline{<} \frac{--\geq}{\text { i0 }}$ Selid $\stackrel{\text { selid }}{\longrightarrow}$ Term \\
\hline binary product & $\frac{Y_{1} Y_{2}}{p r_{i}: Y_{1} \times Y_{2} \rightarrow Y_{i}} i=1,2$ & Type $^{2}<\frac{--\geq}{j} 2$-Prod $\stackrel{2-\text { prod }}{\longrightarrow} 2$-Cone \\
\hline binary tuple & $\frac{f_{1}: X \rightarrow Y_{1} f_{2}: X \rightarrow Y_{2}}{\left\langle f_{1}, f_{2}\right\rangle: X \rightarrow Y_{1} \times Y_{2}}$ & 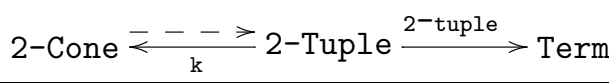 \\
\hline terminal type & $\overline{1}$ & Unit $\underset{\text { j0 }}{\longleftarrow} 0$-Prod $\stackrel{0-\text { prod }}{\longrightarrow}$ Type \\
\hline collapsing & $\frac{X}{\langle\rangle_{X}: X \rightarrow 1}$ & 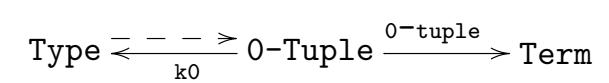 \\
\hline
\end{tabular}

Figure 2: Rules for the equational logic

The theories of the equational logic with a parameter $L_{a}$ are the parameterized equational theories together with a distinguished constant of type $A$, called the parameter and usually denoted $a: 1 \rightarrow A$. The specifications are the parameterized equational specifications with maybe a distinguished term $a: 1 \rightarrow A$. The inclusion of limit sketches determines a morphism of logics $F_{a}: L_{A} \rightarrow L_{a}$.

The theories of the decorated equational logic $L_{d e c}$ are the equational theories together with a wide subtheory called pure (wide means with the same types). The specifications are the equational specifications together with a wide subspecification. Here is a way to build $\mathbf{E}_{d e c, T}$ from $\mathbf{E}_{e q, T}$ which reflects the meaning of the word "decoration", a smaller choice for $\mathbf{E}_{d e c, T}$ can be found in Domínguez and Duval 2009. The decorations in this context are simply made of two keywords $p$ for "pure" and $g$ for "general"; some terms are pure, all terms are general, and there are rules for dealing with the decorations: identities and projections are always pure, and the compositions or tuples of pure terms are pure. This information can be encoded as a realization $\Delta$ of $\mathbf{E}_{e q, T}$ with values in the category of equational theories, as follows. First let us describe the set-valued realization $\Delta_{0}$ of $\mathbf{E}_{e q, T}$ underlying $\Delta$. The set $\Delta_{0}$ (Type) is made of one type $D$ and the set $\Delta_{0}$ (Term) of two terms $p$ and $g$, so that $\Delta_{0}$ (Cons) $=\{(p, p),(p, g),(g, p),(g, g)\}, \Delta_{0}(2$-Cone $)=$ $\{(p, p),(p, g),(g, p),(g, g)\}$ and $\Delta_{0}\left(\right.$ Type $\left.^{2}\right)=\{(D, D)\}$, and we denote $\Delta_{0}($ Unit $)=\{\star\}$. Then $\Delta_{0}($ selid $)$ maps $D$ to $p, \Delta_{0}$ (comp) maps $(p, p)$ to $p$ and everything else to $g, \Delta_{0}$ (2-prod) maps $(D, D)$ to $(p, p)$, $\Delta_{0}$ (2-tuple) maps $(p, p)$ to $p$ and everything else to $g, \Delta_{0}$ (0-prod) maps $\star$ to $p$ and $\Delta_{0}(0$-tuple) maps $D$ to $p$. The structure of equational theory on each set $\Delta_{0}(E)$ is induced by a monomorphism $p \rightarrow g$ in $\Delta($ Term). Then $\mathbf{E}_{d e c, T}$ is the sketch of elements (similar to the more usual category of elements) of the realization $\Delta$ of $\mathbf{E}_{e q, T}$ : the points of $\mathbf{E}_{d e c, T}$ include one point Type.D over the point Type of $\mathbf{E}_{e q, T}$, two points Term.p and Term.g over the point Term of $\mathbf{E}_{e q, T}$, four points over Cons, and so on, and the arrows of $\mathbf{E}_{d e c, T}$ include an arrow c: Term.p $\rightarrow$ Term.g over id $_{\text {Term }}$ which is a potential monomorphism, for the conversion of pure terms to general terms.

Clearly by forgotting the decorations we get a morphism of diagrammatic logics $F_{u n d}: L_{d e c} \rightarrow L_{e q}$, called the undecoration morphism. And by mapping every feature of $\mathbf{E}_{e q, T}$ to the corresponding pure feature of $\mathbf{E}_{d e c, T}$ we get a morphism of diagrammatic logics $F_{p}: L_{e q} \rightarrow L_{d e c}$ such that $F_{u n d} \circ F_{p}=i d_{L_{e q}}$.

\subsection{The parameterization process is a morphism of logics}

In this section we define a morphism of logics $F_{p a r}: L_{d e c} \rightarrow L_{A}$. We define $F_{p a r}$ on specifications, its definition on theories follows easily. We will use the fact, which follows from the definition of a morphism of logics, 
that a specification may be replaced by an equivalent one whenever needed.

The parameterization process starts from a decorated specification and returns a parameterized specification. Roughly speaking, it replaces every general feature in a decorated specification by a parameterized one, in such a way that a pure feature does not really depend on the parameter. More precisely, types and pure terms are unchanged, while every general term $f: X \rightarrow Y$ is replaced by $f^{\prime}: A \times X \rightarrow Y$ where $A$ is the type of parameter. Figure 3 defines the image of the elementary decorated specifications (pure terms are denoted with " $\rightsquigarrow$ " and the projections $p r_{X}: A \times X \rightarrow A$ and $\varepsilon_{X}: A \times X \rightarrow X$ are often omitted): for each point E.x in $\mathbf{E}_{d e c, S}$, the parameterization process replaces the elementary decorated specification $\mathcal{Y}($ E.x $)$ by the parameterized specification $F_{\text {par }}(\mathcal{Y}($ E.x $))$. The morphisms between elementary decorated specifications are transformed in a straightforward way. For instance, the image of the morphism $\mathcal{Y}(\mathrm{c})$, where c: Term.p $\rightarrow$ Term.g is the conversion arrow, maps $f^{\prime}: A \times X \rightarrow X$ in $F_{\text {par }}\left(\mathcal{Y}\right.$ (Term.g)) to $f \circ \varepsilon_{X}: A \times X \rightarrow Y$ in $F_{\text {par }}\left(\mathcal{Y}\left(\right.\right.$ Term.p)), or more precisely in a parameterized specification equivalent to $F_{\text {par }}(\mathcal{Y}($ Term.p)). This provides a graph morphism $F_{\text {par }}: \operatorname{Real}_{e l}\left(\mathbf{E}_{d e c, S}\right) \rightarrow \operatorname{Real}\left(\mathbf{E}_{A, S}\right)$.

\begin{tabular}{|c|c|c|c|}
\hline & point E.x & $\mathcal{Y}(\mathrm{E} . \mathrm{x})$ & $F_{p a r}(\mathcal{Y}($ E.x $))$ \\
\hline type & Type.p & $X$ & $X$ \\
\hline pure term & Term.p & $X \sim \stackrel{f}{\sim} \sim Y$ & $X \stackrel{f}{\longrightarrow} Y$ \\
\hline term & Term.g & $X \stackrel{f}{\longrightarrow} Y$ & $A \times X \stackrel{f^{\prime}}{\longrightarrow} Y$ \\
\hline pure composition & Comp.p & 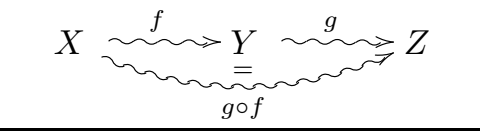 & 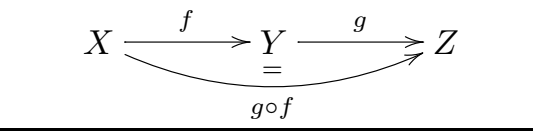 \\
\hline composition & Comp.g & 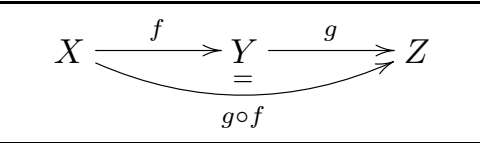 & $A \times X \underset{=}{\stackrel{\left\langle p r_{X}, f^{\prime}\right\rangle}{\longrightarrow} \underset{g^{\prime} \circ\left\langle p r_{X}, f^{\prime}\right\rangle}{A} \stackrel{g^{\prime}}{\longrightarrow} Z}$ \\
\hline selection of identity & Selid.p & $X \stackrel{i d_{X}}{\sim} \longrightarrow X$ & $X \stackrel{i d_{X}}{\longrightarrow} X$ \\
\hline binary product & 2-Prod.p & 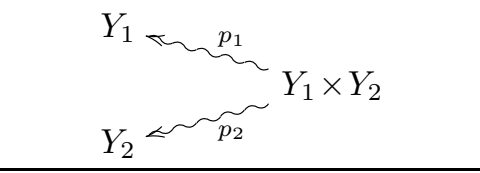 & $\begin{array}{l}Y_{1} \\
Y_{2}\end{array}$ \\
\hline pure pairing & 2-Tuple.p & 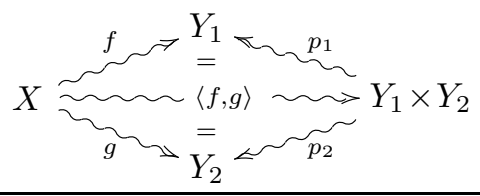 & 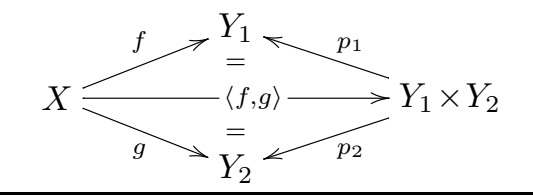 \\
\hline pairing & 2-Tuple.g & 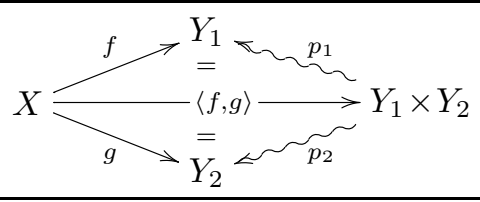 & 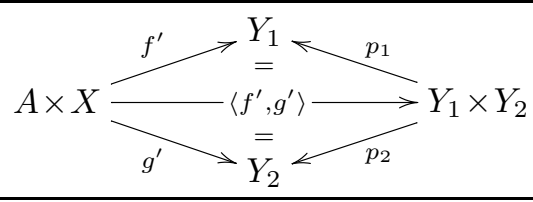 \\
\hline terminal type & 0-Prod.p & 1 & 1 \\
\hline pure collapsing & 0-Tuple.p & $X \stackrel{\langle\rangle_{X}}{\sim}>1$ & $X \stackrel{\langle\rangle_{X}}{\longrightarrow} 1$ \\
\hline
\end{tabular}

Figure 3: The parameterization morphism on elementary decorated specifications 
Theorem 3.1 The graph morphism $F_{\text {par }}$ defines a morphism of diagrammatic logics:

$$
F_{p a r}: L_{d e c} \rightarrow L_{A}
$$

which is the inclusion on the pure part of $L_{d e c}$, in the sense that $F_{p a r} \circ F_{p}=F_{A}$. It is called the parameterization morphism.

Proof. It can be checked that this graph morphism preserves the distinguished features of $\operatorname{Real}_{e l}\left(\mathbf{E}_{d e c, S}\right)$ and the entailments of the decorated logic, so that it provides a morphism of diagrammatic logics. The equality $F_{p a r} \circ F_{p}=F_{A}$ is easily checked on elementary specifications.

The morphisms of logics $F_{u n d}, F_{p a r}$ and $F_{A}$ form a (non-commutative) triangle, which becomes commutative when restricted to the pure part of $L_{d e c}$ :

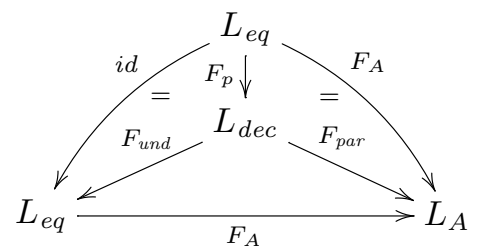

The parameterization morphism $F_{p a r}$ formalizes the parameterization process. The span made of $F_{\text {und }}$ and $F_{p a r}$ formalizes the process of starting from an equational specification $\Sigma_{e q}$, choosing a pure subspecification $\Sigma_{0}$ of $\Sigma_{e q}$ so as to get a decorated specification $\Sigma_{d e c}$ such that $\Sigma_{e q}=F_{\text {und }}\left(\Sigma_{d e c}\right)$, then forming the parameterized specification $\Sigma_{A}=F_{p a r}\left(\Sigma_{d e c}\right)$.

\subsection{The parameter passing process is a 2-morphism of logics}

The diagram of logics in section 3.2 composed with the inclusion $F_{a}: L_{A} \rightarrow L_{a}$, which adds the parameter $a: 1 \rightarrow A$, provides another diagram with in addition a 2 -morphism $\ell$ as described below:

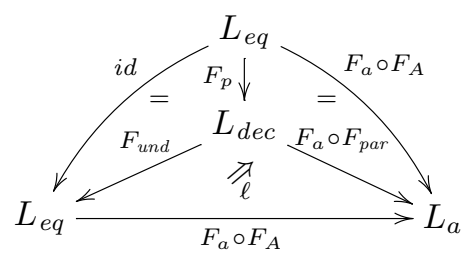

Each decorated specification $\Sigma_{d e c}$, with $\Sigma_{e q}=F_{u n d}\left(\Sigma_{d e c}\right)$, gives rise to two specifications with parameter: on the one hand $\Sigma_{e q, a}=F_{a}\left(F_{A}\left(\Sigma_{e q}\right)\right)$, which is simply $\Sigma_{e q}$ seen as a specification with a parameter, and on the other hand $\Sigma_{a}=F_{a}\left(F_{p a r}\left(\Sigma_{d e c}\right)\right)$. Let us define the morphism $\ell_{\Sigma_{d e c}}: \Sigma_{e q, a} \rightarrow \Sigma_{a}$. When $\Sigma_{d e c}$ is some $\mathcal{Y}$ (E.p) (where p means "pure") it is easy to check that $\Sigma_{e q, a}=\Sigma_{a}$; then $\ell_{\Sigma_{\text {dec }}}$ is the identity. When $\Sigma_{\text {dec }}=$ $\mathcal{Y}_{\text {dec }}$ (Term.g) (where g means "general"), then $\ell_{\Sigma_{d e c}}$ is defined by $\ell_{\Sigma_{d e c}}(f)=f^{\prime} \circ\left\langle a, i d_{X}\right\rangle: X \rightarrow Y$ (where $1 \times X$ is identified with $X$ ). The definitions when $\Sigma_{d e c}=\mathcal{Y}_{d e c}$ (Comp.g) and when $\Sigma_{d e c}=\mathcal{Y}_{d e c}$ (2-Tuple.g) are similar.

Theorem 3.2 The morphisms $\ell_{\Sigma_{d e c}}: \Sigma_{e q, a} \rightarrow \Sigma_{a}$ define a 2-morphism of diagrammatic logics:

$$
\ell: F_{a} \circ F_{A} \circ F_{\text {und }} \Rightarrow F_{a} \circ F_{\text {par }}: L_{d e c} \rightarrow L_{a}
$$

which is the identity on the pure part of $L_{d e c}$. It is called the parameter passing 2-morphism.

Proof. The definition of $\ell_{\Sigma_{d e c}}$ on the elementary decorated specifications is extended to all specifications by colimits, and the result follows.

Theorem 3.2 has the expected consequence on models, stated as proposition 3.3: given a set-valued model $M_{A}$ of the paramererized specification $\Sigma_{A}$, each $\alpha \in M_{A}(A)$, called an actual parameter or an argument, 
gives rise to a model $\mathcal{M}(\alpha)$ of the equational specification $\Sigma_{e q}$. Let us introduce some notations. For each set $\mathbb{A}$, let $S e t_{\mathbb{A}}$ denote the object of $\mathbf{T}_{A}$ made of the equational theory of sets with $\mathbb{A}$ as the interpretation of $A$, so that $R_{A}\left(\operatorname{Set}_{\mathbb{A}}\right)=$ Set. For each set $\mathbb{A}$ and element $\alpha \in \mathbb{A}$, let $\operatorname{Set}_{\mathbb{A}, \alpha}$ denote the object of $\mathbf{T}_{a}$ made of the equational theory of sets with $\mathbb{A}$ and $\alpha$ as the interpretations of $A$ and $a$ respectively, so that $R_{a}\left(\operatorname{Set}_{\mathbb{A}, \alpha}\right)=\operatorname{Set}_{\mathbb{A}}$. For each decorated specification $\Sigma_{\text {dec }}=\left(\Sigma_{e q}, \Sigma_{0}\right)$, made of an equational specification $\Sigma_{e q}$ and a wide subspecification $\Sigma_{0}$, and for each set-valued equational model $M_{0}$ of $\Sigma_{0}$, let $\left.L_{e q}\left[\Sigma_{e q}, S e t\right]\right|_{M_{0}}$ denote the set of models of $\Sigma_{e q}$ extending $M_{0}$. Let $\Sigma_{A}=F_{p a r}\left(\Sigma_{d e c}\right)$, the definition of $F_{p a r}$ is such that $\Sigma_{0}$ is also a subspecification of $\Sigma_{A}$ and for each $f: X \rightarrow Y$ in $\Sigma_{e q}$ there is a $f^{\prime}: A \times X \rightarrow Y$ in $\Sigma_{A}$, with $f^{\prime}=f \circ \varepsilon_{X}$ when $f$ is pure.

Proposition 3.3 Let $\Sigma_{d e c}=\left(\Sigma_{e q}, \Sigma_{0}\right)$ be a decorated specification and let $\Sigma_{A}=F_{\text {par }}\left(\Sigma_{\text {dec }}\right)$. For each set $\mathbb{A}$ and each set-valued model $M_{A}: \Sigma_{A} \rightarrow$ Set ${ }_{\mathbb{A}}$ in $L_{A}$, let $M_{0}: \Sigma_{e q} \rightarrow$ Set denote the restriction of $M_{A}$ to $\Sigma_{0}$. Then there is a function:

$$
\mathcal{M}:\left.\mathbb{A} \rightarrow L_{e q}\left[\Sigma_{e q}, \text { Set }\right]\right|_{M_{0}}
$$

which maps each $\alpha \in \mathbb{A}$ to the model $\mathcal{M}(\alpha)$ of $\Sigma_{e q}$ extending $M_{0}$ and such that $\mathcal{M}(\alpha)(f)=M_{A}\left(f^{\prime}\right)(\alpha,-)$ for each $f: X \rightarrow Y$ in $\Sigma_{e q}$.

Proof. Let $\Sigma_{e q, a}=F_{a}\left(F_{A}\left(\Sigma_{e q}\right)\right)$ and $\Sigma_{a}=F_{a}\left(F_{\text {par }}(\Sigma d e c)\right)$. The precomposition with the morphism $\ell_{\Sigma_{d e c}}: \Sigma_{e q, a} \rightarrow \Sigma_{a}$ gives rise to a functor $L_{a}\left[\Sigma_{a}, S e t_{\mathbb{A}, \alpha}\right] \rightarrow L_{a}\left[\Sigma_{e q, a}, S_{e} t_{\mathbb{A}, \alpha}\right]$. Proposition 2.11 provides the isomorphisms $L_{a}\left[\Sigma_{a}, S e t_{\mathbb{A}, \alpha}\right] \cong L_{A}\left[\Sigma_{A}, \operatorname{Set}_{\mathbb{A}}\right]$ and $L_{a}\left[\Sigma_{e q, a}, \operatorname{Set}_{\mathbb{A}, \alpha}\right] \cong L_{e q}\left[\Sigma_{e q}\right.$, Set $]$. So, for each $\alpha \in \mathbb{A}$ we get a functor $L_{A}\left[\Sigma_{A}, S e t_{\mathbb{A}}\right] \rightarrow L_{e q}\left[\Sigma_{e q}\right.$, Set]. Let $M_{A, \alpha}$ denote the image of $M_{A}$, because of the definition of $\ell_{\Sigma_{d e c}}$ it extends $M_{0}$ and satisfies $M_{A, \alpha}(f)=M_{A}\left(f^{\prime}\right)(\alpha,-)$ for each $f: X \rightarrow Y$ in $\Sigma_{e q}$. Now, when $M_{A}$ is fixed, the result follows by defining $\mathcal{M}(\alpha)=M_{A, \alpha}$.

The function $\mathcal{M}$ is not a bijection in general. However this may happen, under the conditions of proposition 3.4: this is the exact parameterization property from Lambán et al. 2003], which is also proved in [Domínguez and Duval 2009].

Proposition 3.4 With the specifications $\Sigma_{e q}, \Sigma_{0}$ and $\Sigma_{A}$ as in proposition 3.9, let $M_{0}$ be a model of $\Sigma_{0}$ and $M_{A}$ a terminal model of $\Sigma_{A}$ extending $M_{0}$. Then the function $\mathcal{M}$ from proposition 3.3 is a bijection:

$$
\left.M_{A}(A) \cong L_{e q}\left[\Sigma_{e q}, S e t\right]\right|_{M_{0}} .
$$

It follows from Rutten 2000 and Hensel and Reichel 1995 that there is a terminal model of $\Sigma_{A}$ over $M_{0}$. Proposition 3.4 corresponds to the way algebraic structures are implemented in the systems Kenzo/EAT. In these systems the parameter set is encoded by means of a record of Common Lisp functions, which has a field for each operation in the algebraic structure to be implemented. The pure terms correspond to functions which can be obtained from the fixed data and do not require an explicit storage. Then, each particular instance of the record gives rise to an algebraic structure.

\section{References}

[Barr and Wells 1999] Barr, M. and Wells, C. (1999) Category Theory for Computing Science. Centre de Recherches Mathématiques (CRM) Publications, 3rd Edition.

[Coppey and Lair 1984] Coppey, L. and Lair, C. (1984) Leçons de Théorie des Esquisses. Diagrammes 12.

[Domínguez and Duval 2009] Domínguez, C. and Duval, D. (2009) A parameterization process as a categorical construction.

[Domínguez et al. 2005] Domínguez, C., Duval, D., Lambán, L. and Rubio, J. (2005) Towards diagrammatic specifications of symbolic computation systems. In: Mathematics, Algorithms, Proofs. Coquand, T., Lombardi, H. and Roy, M. (Eds.) Dagstuhl Seminar 05021. http://drops.dagstuhl.de/portals/index. php?semnr=05021. 
[Domínguez et al. 2007] Domínguez, C., Lambán, L. and Rubio, J. (2007) Object-oriented institutions to specify symbolic computation systems. Rairo - Theoretical Informatics and Applications 41 191-214.

[Domínguez et al. 2006] Domínguez, C., Rubio, J. and Sergeraert, F. (2006) Modeling Inheritance as coercion in the Kenzo system. Journal of Universal Computer Science 12 (12) 1701-1730.

[Dousson et al. 1999] Dousson, X., Sergeraert, F. and Siret, Y. (1999) The Kenzo program. Institut Fourier, Grenoble. http://www-fourier.ujf-grenoble.fr/ sergerar/Kenzo.

[Dumas et al. 2009] Dumas, J.G., Duval, D. and Reynaud, J.C. (2009) Cartesian effect categories are Freydcategories. arXiv:0903.3311v3.

[Duval 2003] Duval, D. (2003) Diagrammatic specifications. Mathematical Structures in Computer Science 13 857-890.

[Duval 2007] Duval, D. (2007) Diagrammatic inference. arXiv:0710.1208v1.

[Ehresmann 1968] Ehresmann, C. (1968) Esquisses et types de structures algébriques. Bull. Instit. Polit. Iaşi XIV.

[Gabriel and Ulmer 1971] Gabriel, P. and Ulmer, F. (1971) Lokal präsentierbare Kategorien. Springer Lecture Notes in Mathematics 221.

[Gabriel and Zisman 1967] Gabriel, P. and Zisman, M. (1967) Calculus of Fractions and Homotopy Theory. Springer.

[Goguen and Burstall 1984] Goguen, J. A. and Burstall, R. M. (1984) Introducing Institutions. Springer Lecture Notes in Computer Science 164 221-256.

[Goguen and Malcolm 2000] Goguen, J. and Malcolm, G. (2000) A hidden agenda. Theoretical Computer Science 245 (1) 55-101.

[Hensel and Reichel 1995] Hensel, U. and Reichel, H. (1995) Defining equations in terminal coalgebras. In: Recent Trends in Data Type Specifications, Springer Lecture Notes in Computer Science 906 307-318.

[Kan 1958] Kan, D.M. (1958) Adjoint Functors. Transactions of the American Mathematical Society 87 294-329.

[Lambán et al. 2003] Lambán, L., Pascual, V. and Rubio, J. (2003) An object-oriented interpretation of the EAT system. Applicable Algebra in Engineering, Communication and Computing 14 (3) 187-215.

[Lellahi 1989] Lellahi, S.K. (1989) Categorical abstract data type (CADT). Diagrammes 21, SKL1-SKL23.

[Mac Lane 1998] Mac Lane, S. (1998) Categories for the Working Mathematician. Springer, 2 th edition.

[Makkai 1997] Makkai, M. (1997) Generalized sketches as a framework for completeness theorems (I). Journal of Pure and Applied Algebra 115 49-79.

[Pitts 2000] Pitts, A.M. (2000) Categorical Logic. Chapter 2 of Abramsky, S., Gabbay, D.M. and Maibaum, T.S.E. (Eds.) Handbook of Logic in Computer Science 5. Algebraic and Logical Structures. Oxford University Press.

[Rubio et al. 2007] Rubio, J., Sergeraert, F. and Siret, Y. (2007) EAT: Symbolic Software for Effective Homology Computation. Institut Fourier, Grenoble. ftp://fourier.ujf-grenoble.fr/pub/EAT.

[Rutten 2000] Rutten, J.J.M.M. (2000) Universal coalgebra: a theory of systems. Theoretical Computer Science 249 (1) 3-80.

[Wells 1993] Wells, C. (1993) Sketches: Outline with References. http://www.cwru.edu/artsci/math/ wells/pub/papers.html. 